\title{
Multifactorial Approaches for Correction of the Drooping Tip of a Long Nose in East Asians
}

\author{
Seong Geun Park, Hoijoon Jeong, Choon Ho Ye \\ Joseph's Plastic Surgery Center, Daegu, Korea
}

\begin{abstract}
A long nose with a drooping tip is a major aesthetic problem. It creates a negative and aged appearance and looks worse when smiling. In order to rectify this problem, the underlying anatomical causes should be understood and corrected simultaneously to optimize surgical outcomes. The causes of a drooping tip of a long nose are generally classified into two mechanisms. Static causes usually result from malposition and incorrect innate shape of the nasal structure: the nasal septum, upper and lower lateral cartilages, and the ligaments in between. The dynamic causes result from the facial expression muscles, the depressor septi nasi muscle, and the levator labii superioris alaeque nasi muscle. The depressor septi nasi depresses the nasal tip and the levator labii superioris alaeque nasi pulls the alar base upwards. Many surgical methods have been introduced, but partial approaches to correct such deformities generally do not satisfy East Asians, making the problem more challenging to surgeons. Typically, East Asians have thick nasal tip soft tissue and skin, and a depressed columella and alar bases. The authors suggest that multifactorial approaches to static and dynamic factors along with ancillary causes should be considered for correcting the drooping tip of the long noses of East Asians.
\end{abstract}

Keywords: Esthetics / Nose / Rhinoplasty

\author{
Correspondence: Seong Geun Park \\ Joseph's Plastic Surgery Center, 99 \\ Dongseong-ro 3-gil, Jung-gu, \\ Daegu 700-150, Korea \\ Tel: $+82-53-431-3388$ \\ Fax: +82-53-422-9100 \\ E-mail: carmelpark@hanmail.net
}

No potential conflict of interest relevant to this article was reported.

Received: 24 Jul 2013 • Revised: 9 Sep 2013 • Accepted: 10 Sep 2013

pISSN: 2234-6163 • elSSN: 2234-6171 • http://dx.doi.org/10.5999/aps.2014.41.6.630 • Arch Plast Surg 2014;41:630-637

\section{INTRODUCTION}

The ideal length of the nose is two-thirds of the distance from the glabella to the alar base plane; this should be the same as the distance from the stomion to the mentum [1]. However, the ideal nasal length is subjective, and varies with respect to patient characteristics, the width of the face, and the correlation with the lips and the chin. Therefore, the suggested ideal length should not always be the aim of the correction; rather, the preference of the patient is of greater consideration.

In general, long noses have the following characteristics: 1 ) a drooping tip and caudal displacement of the nasal tip, 2) a large mobile tip (lower third of the nose), 3) an acute columella-labial angle, 4) a nasal dorsal hump and convex nasal dorsum, 5) an absent or shallow nasofrontal angle, and 6) thick sebaceous skin. The underlying anatomical causes of such characteristics should be understood and all of them should be corrected at the same time to optimize the outcomes of the surgery.

In particular, the drooping tip phenomenon causes long-looking noses and an aged facial appearance. In the case of a hump nose, it forms a negative image, like a witch's nose or a Captain Hook's nose. When smiling, the facial expression muscles worsen the drooping tip. Moreover, East Asians tend to have thicker skin than Caucasians and have underdeveloped alar cartilages. 
This makes the correction even more difficult for surgeons, as the results are not generally satisfying. Therefore, a comprehensive analysis of the anatomical structures and surgical methods should be considered.

Herein, we present our experience with the correction of long noses with a particular emphasis on correcting the drooping tip, and review the relevant literature.

\section{ANATOMICAL CAUSES OF A DROOPING TIP}

The causes of a drooping tip can be divided into problems of the alar cartilage, septal cartilage (static causes), and the facial expression muscles (dynamic causes). In most cases, all of these contribute to the drooping tip.

\section{Static problems of alar cartilage and septal cartilage}

1) Excessively long and vertically oriented lateral crura and/or caudally dislocated alar cartilage with a weak aponeurotic attachment to the anterior septal angle.

2) Long caudal septum with/without a highly positioned anterior septal angle.

\section{Dynamic pulling forces on the nasal tip by underlying muscles}

Dynamic forces that cause a drooping tip are generated by the following two muscles [2]: 1) Depressor septi nasi muscle: This is a small muscle connecting the tip to the bilateral sides of the anterior nasal spine and orbicularis oris muscle. It pulls the tip downwards while smiling [3,4].2) Levator labii superioris alaeque nasi muscle: This muscle pulls the alar base to the cephalic direction on the nasal wing and makes the tip droop more.

These two muscles act as a functional unit to make the drooping tip more prominent and cause recurrence of the drooping tip after surgery (Fig. 1).

\section{CORRECTION OF DROOPING TIP}

For building and maintaining adequate tip projection, the surgeon should simultaneously correct the multiple factors that cause the drooping tip. The following four factors should be kept in mind to surgically correct the drooping tip. First, the alar cartilage or nasal septal cartilage problem should be corrected. Second, to make the corrected alar cartilage stable, that is, to maintain tip projection and columellar shape, a columellar strut or batten graft should be performed. Third, dynamic factors such as those caused by the depressor septi nasi muscle and the levator labii superioris alaeque nasi muscle should be cut or
Fig. 1. Static and dynamic causes of drooping tip in long noses

(A) Static causes usually result from large and long alar cartilages with the distal septum placed downward. (B) Dynamic forces of the depressor septi nasi muscle and levator labii superioris alaeque nasi muscle worsen the drooping tip when smiling.
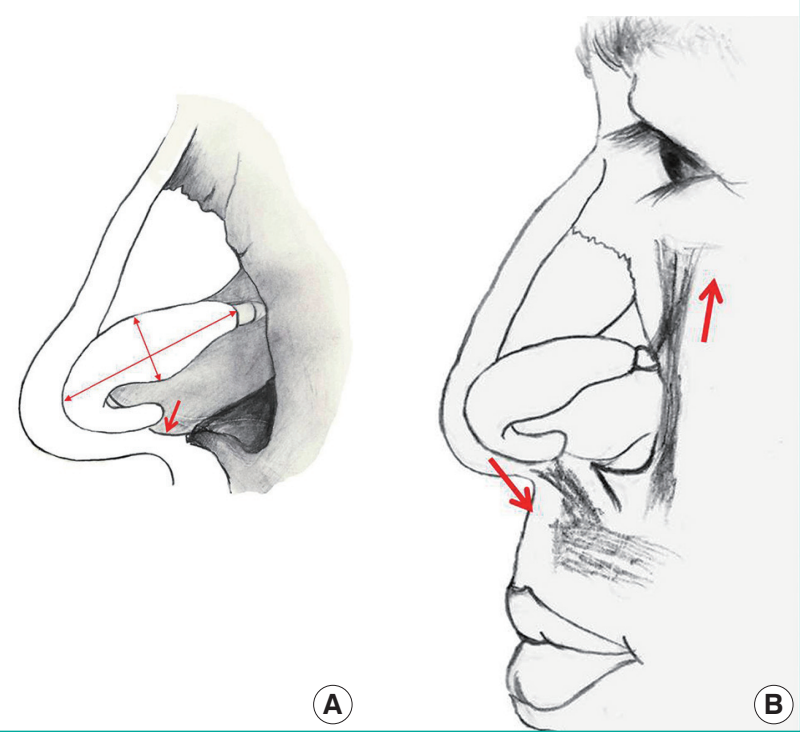

weakened to prevent recurrence. Fourth, in cases with thick tip skin and soft tissue, the redundant tissue before framework modification should be excised.

An algorithm for surgical correction of the drooping tip is outlined (Fig. 2).

\section{Modification of alar and septal cartilage}

Horizontal mattress suture on the proximal portion of the lateral crus for medial crus lengthening: lateral crus stealing technique

In cases with a long longitudinal axis of the lateral crus, and with short, underdeveloped medial crus, a horizontal mattress suture should be performed in the proximal portion of the lateral crus to simultaneously correct the drooping tip and the hanging columella. Depending on the suture site and angle, the nasal tip location changes and the lateral crus advances medially (lateral crus stealing technique) [5]. Narrowing the angle between the medial and the lateral crus of the alar cartilage rotates the tip up and pulls up the hanging columella $[6,7]$. To prevent recurrence, scoring incisions on the perichondrium of the newly created domes are carried out by using a no. 15 blade. Newly located domes should be fixed to the columellar strut or the septal batten graft by a horizontal mattress suture to maintain the new tip shape (Fig. 3). 
Distal septal cartilage excision and columella septal suture

This technique helps in cases of a hanging columella. The medial crura of the alar cartilage should be sutured upward to the caudal septum to simultaneously control the tip height and cor- rect the hanging columella. However, over time, the result might be unsatisfying due to the cheese cutter effect of the suture; therefore, overcorrection should be considered. In cases of an excessively long nasal septum, the distal septal portion should

\section{Fig. 2. Algorithm for surgical correction of drooping tip}

To correct the drooping tip, first, five basic procedures for building and maintaining adequate tip projection are performed, and then additional procedures can be considered. SMAS, superficial musculoaponeurotic system; ANS, anterior nasal spine.
Building and maintaining adequate tip projection (five basic procedures):

1) Horizontal mattress suture on proximal portion of lateral crus for medial crus lengthening (lateral crus stealing technique)

2) The turn up cartilage flap of cephalic portion of lateral crura

3) Floating or fixed type columellar strut graft depending on the development of medial crura

4) Tip graft for more defined tip shape

5) Excision of the depressor septi nasi muscle

Consider additional procedures:

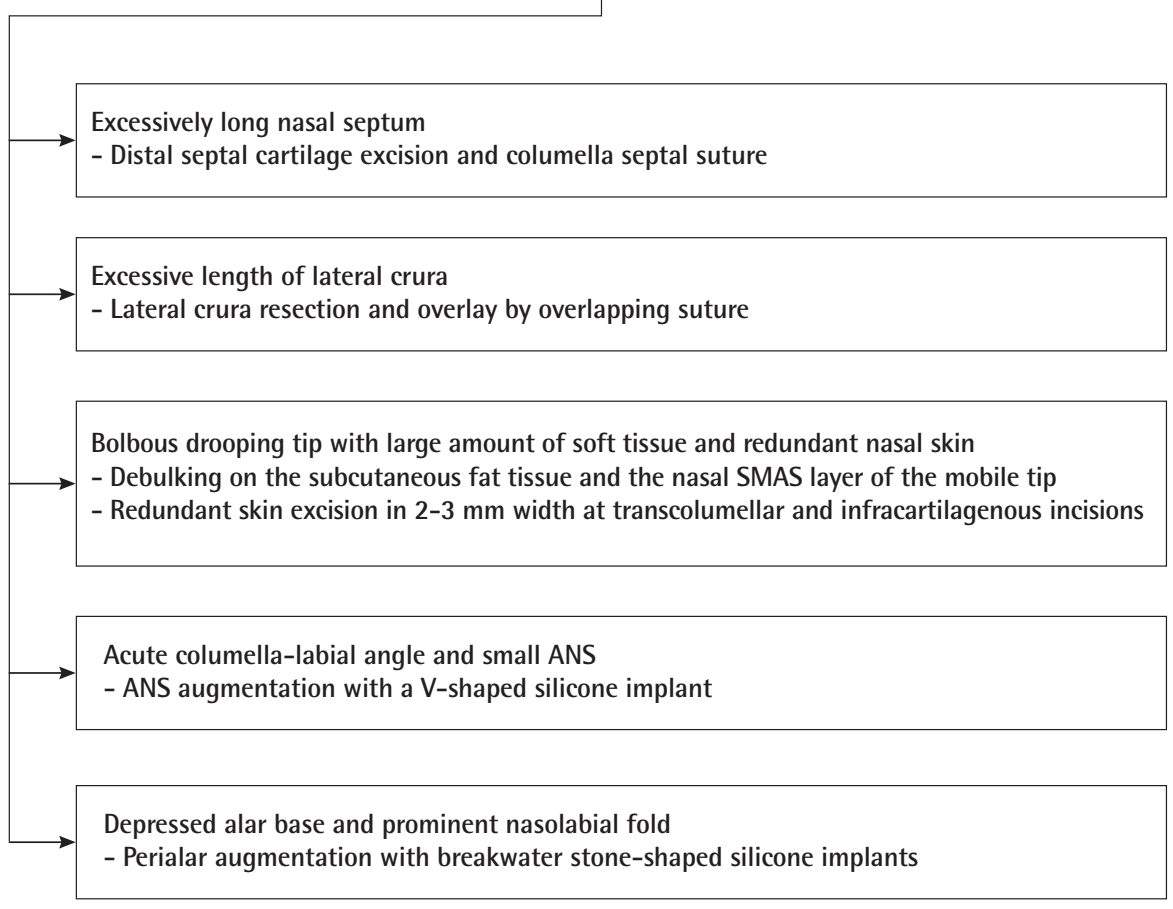

\section{Fig. 3. Lateral crus stealing technique}

A proximal portion of the lateral crus (red line) is advanced medially by a horizontal mattress suture, resulting in tip projection and upward rotation by tip gathering on the columellar strut.

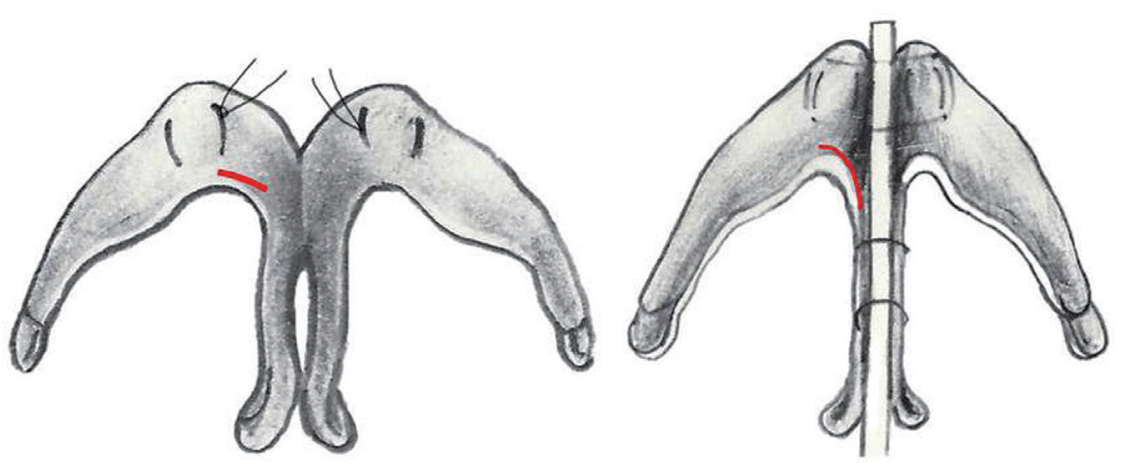


be removed before the suture (Fig. 4).

Turn-up cartilage flap of cephalic portion of lateral crura This technique is especially effective in a relatively large and wide lateral crus. Each cephalic portion of the lateral crura is divided to make a cartilage flap with the pedicle base located on the domal portion of alar cartilage. Then, the flap is attached to

\section{Fig. 4. Correction of a hanging columella}

In the long septum, the distal part can be excised to create a space for the medial crus to be moved upwards. Further, the batten graft or columellar strut is used for ensuring the stability of the medial crus and for tip projection with upward rotation, and two or three layers of cartilage grafts including onlay tip graft, shield graft, and/ or cap graft are performed for tip definition.

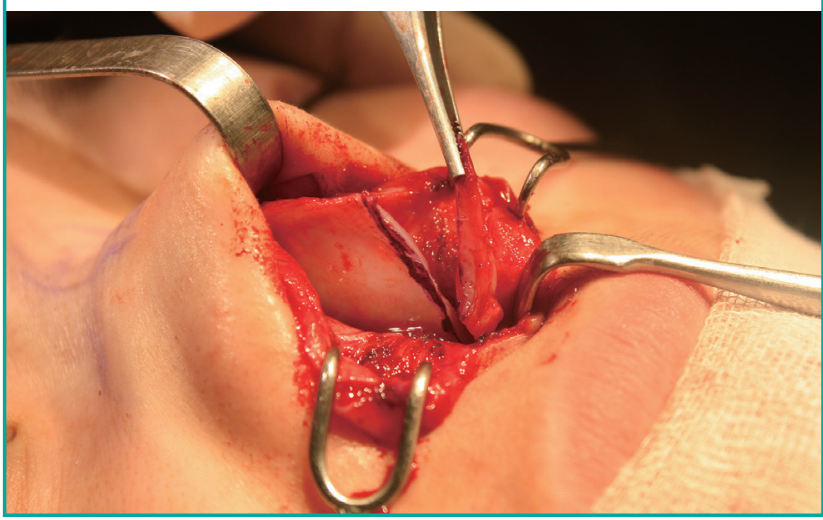

the anterior septal angle to secure the nasal tip in its upward rotation position. The next procedure is to release the fibrous attachment between the upper lateral cartilage and the lower lateral cartilage, making the alar cartilage more cephalically movable. The lateral crural spanning suture used by Dr. Tebbetts [8] helps to narrow the alar width and maintain the tip structure. More volume can be added to the supratip area (Fig. 5). Boccieri [9] attempted to use this flap as a spreader graft, but this is not appropriate for East Asians because of their relatively small alar cartilages.

\section{Lateral crura resection and overlay}

In cases with an excessive length of lateral crura, the distal portion of lateral crura having a width of 3 to $5 \mathrm{~mm}$ could be simply resected or overlaid by the overlapping suture to reduce the length and to make the tip upwardly rotated, according to Dr. Anderson's [10] tripod concept.

\section{Considerations of nasal tip and columellar support Columellar strut graft or batten graft}

In cases of well-developed medial crura, the intercrural suture or floating columellar strut using septal cartilage or auricular cartilage should be used, but in cases with an underdeveloped medial crura, septal cartilage should be used as a fixed-type columellar strut. A columellar strut graft or batten graft clarifies the nasal

\section{Fig. 5. Turn-up cartilage flap}

(A) This picture shows how the cartilage flap is created with the pedicle base located on the domal portion of the alar cartilage. (B) The flap is then attached to the anterior septal angle to secure the nasal tip in its upwardly rotated position.
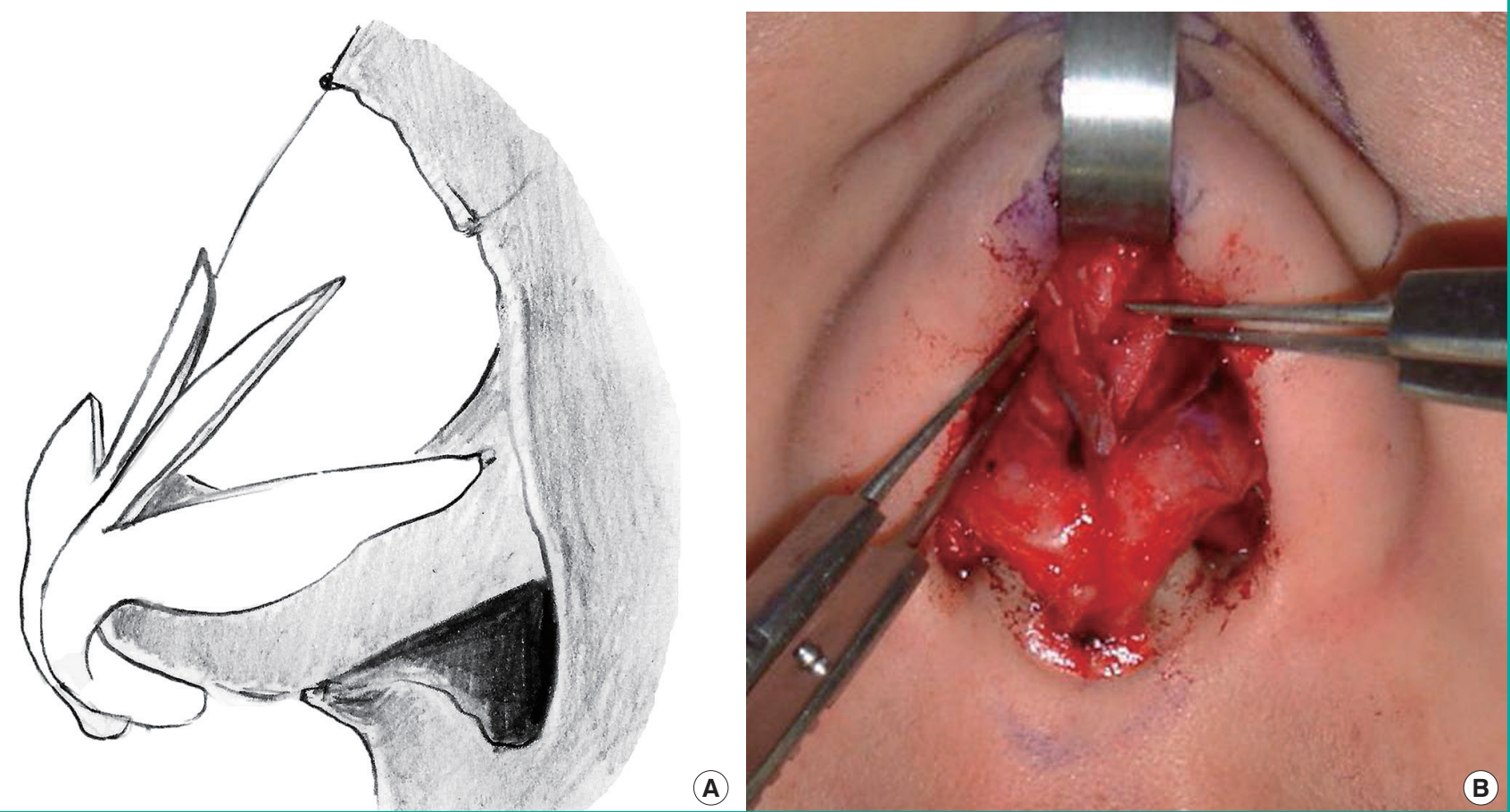
tip definition through an interdomal suture and maintains the position against pulling forces exerted by the depressor septi nasi muscle contraction and skin sutures.

\section{Tip graft}

Thick skin causes unsatisfactory correction of the drooping tip even after appropriate correction of the tip cartilage shape. More defined tips are achieved by multiple methods such as onlay tip graft, shield graft, cap graft, and umbrella graft [11]. The auricular cartilage with perichondrium is used for making a shield graft; it is posteriorly supported by an additional ladder-shaped cartilage graft with the remaining auricular cartilages [12]. This method minimizes the distortion and absorption of the cartilage graft as compared to the pyramidal method.

\section{Solving problems with muscles: dynamic components}

Much research has been published about the depressor septi nasi muscle and the levator labii superioris alaeque nasi muscle, but there is still controversy surrounding the structures and shapes [4]. In particular, in the case of East Asians, their nasal tips are not sufficiently large to allow the surgeon to visualize the muscle actions. In general, the depressor septi nasi muscle pulls the nasal tip downward, and the levator labii superioris alaeque nasi muscle elevates the alar base. The authors suggest that skin traction force, running from both the lateral cheek to the nasal tip along the skin of the nasolabial fold, alar base, and alar rim could cause the drooping when smiling. Therefore, the excision or incision of the muscle and an autogenous graft or artificial implant insertion on the anterior nasal spine and the alar base can be an ancillary solution for the drooping tip.

\section{Fig. 6. Excision of the depressor septi nasi muscle}

The depressor septi nasi muscle between the foot plates of the medial crus and the dermocartilagenous ligament (Pitanguy's ligament) connecting the supratip area should be removed. The excised soft tissue can be rolled and inserted in front of the anterior nasal spine to eliminate the dynamic tip drooping forces and augment the acute columella-labial angle.

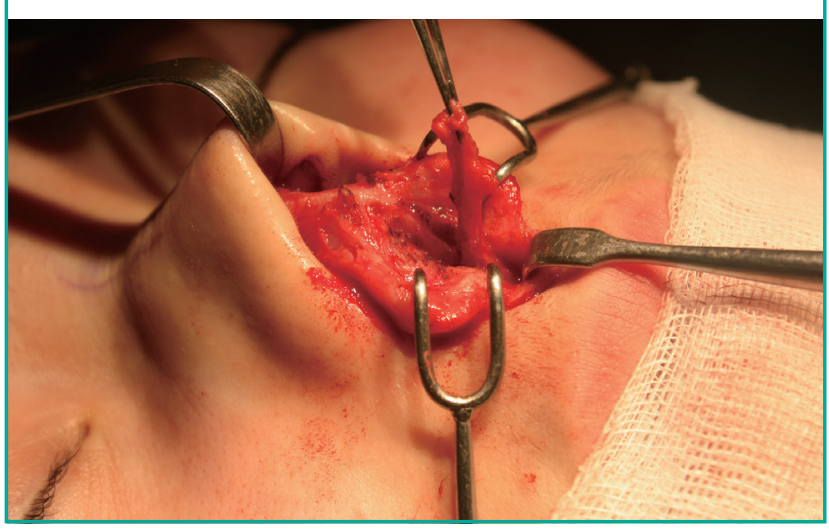

Excision of depressor septi nasi muscle and columellar base augmentation

First, a stepladder-shaped transcolumellar incision is performed. Then, a dissection upward toward the dome, inferiorly toward the anterior nasal spine, and posteriorly toward the septal cartilage should be performed to expose the perichondrium of the medial crus. The depressor septi nasi muscle between the crura or complex tissue should be excised during the dissection, and the excised soft tissue should be rolled and inserted in front of the anterior nasal spine to augment the columella-labial angle (Fig. 6). If the columella-labial angle is too acute and the drooping tip is severe, the origin of the depressor septi nasi muscle should be dissected by using an intraoral approach, and the anterior nasal spine should be exposed to insert a V-shaped silicone implant in the dead space (Fig. 7).

Weakening of the levator labii superioris alaeque nasi muscle and perialar augmentation

If the alar bases are pulled upward too much when one smiles, the nose looks too long and resembles an arrow shape. In cases with depressed alar bases, the nasolabial fold is prominent and causes an aged-looking face; further, the alar base tends to pull upwards to make the drooping tip more noticeable. The levator labii superioris alaeque nasi muscles attached to the lateral crura abutment of the alar cartilage, which is the nasal hinge, causes the pulling [4]. An intraoral incision beside the canine can be performed to dissect the levator labii superioris alaeque nasi muscle in the alar base and to insert a breakwater stone-shaped silicone implant under the periosteal dissection to elevate the alar base (Fig. 8). As a result, the weakened muscular function of the levator labii superioris alaeque nasi muscle decreases the ele-

Fig. 7. Columellar base augmentation using V-shaped implant

The anterior nasal spine is exposed by an intraoral approach, and a V-shaped silicone implant is inserted.

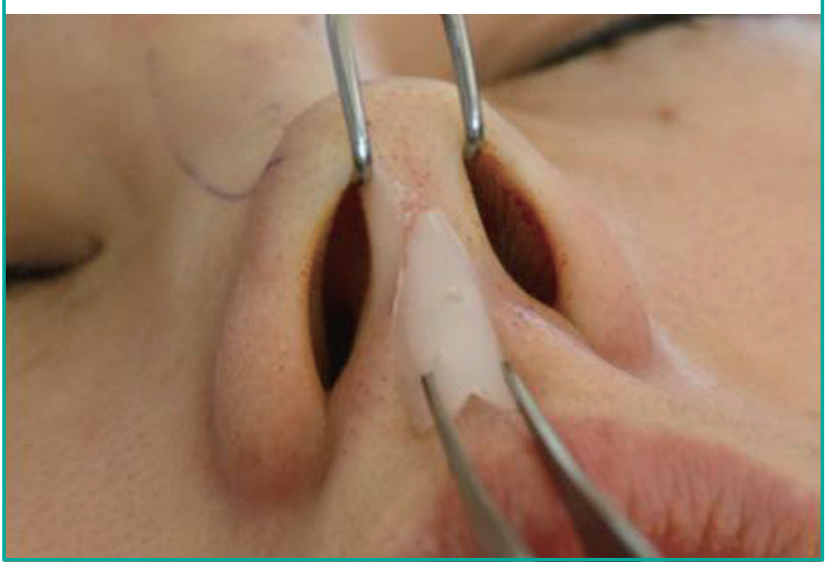


vation of the alar bases when one smiles. Further, the augmentation of the alar base corrects the nasolabial fold to create a better appearance.

\section{Debulking of a bulbous drooping tip and redundant nasal skin management}

Even after appropriate surgical reconstruction of the nasal tip cartilage framework, some soft tissue bulkiness and nasal tip skin redundancy may remain. In patients with soft tissue bulkiness on the nasal tip, debulking was carried out on the subcutaneous fat tissue and the nasal superficial musculoaponeurotic system (SMAS) layer of the mobile tip (Fig. 9). This procedure attaches

\section{Fig. 8. Perialar augmentation using silicone implant}

After the intraoral incision, the levator labii superioris alaeque nasi muscle is dissected in the alar base, and a breakwater stone-shaped silicone implant is inserted under the periosteal dissection to elevate the alar base.

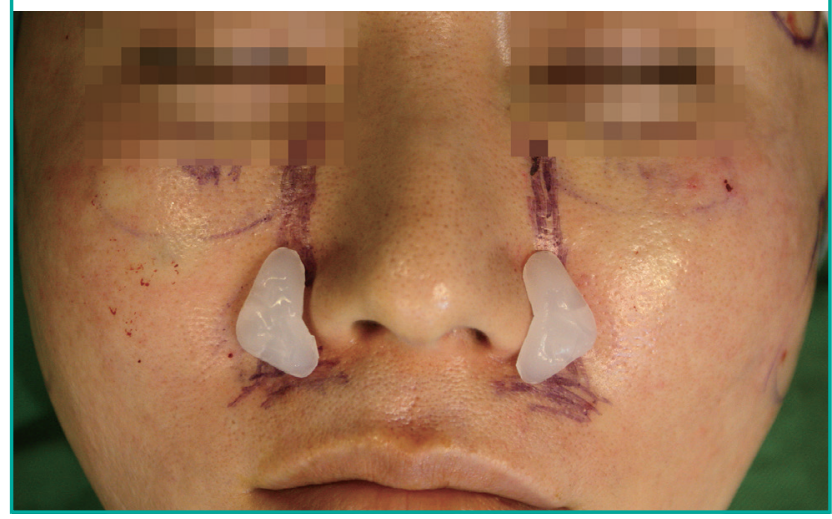

the skin to the cartilage framework and results in the correction of the long and drooping tip caused by soft tissue bulkiness. It also helps the resultant cartilage framework modification to be prominent on the nasal skin layer. Skin contracture following the operation can re-drape the redundant skin to the framework but may require a long time in the case of East Asians who have thick skin, and in some cases, the remaining skin even turns out to make a drooping tip. Therefore, the authors solve the problem by a 2 - to 3-mm-wide excision of redundant skin where the stepladder-shaped transcolumellar incision and infracartilagenous

\section{Fig. 9. Debulking of bulbous drooping tip}

To correct the drooping and bulbous tip and remove the bulky tissues, the redundant fat and the nasal superficial musculoaponeurotic system layer after the subcutaneous dissection should be removed at the supraperichondrial level.

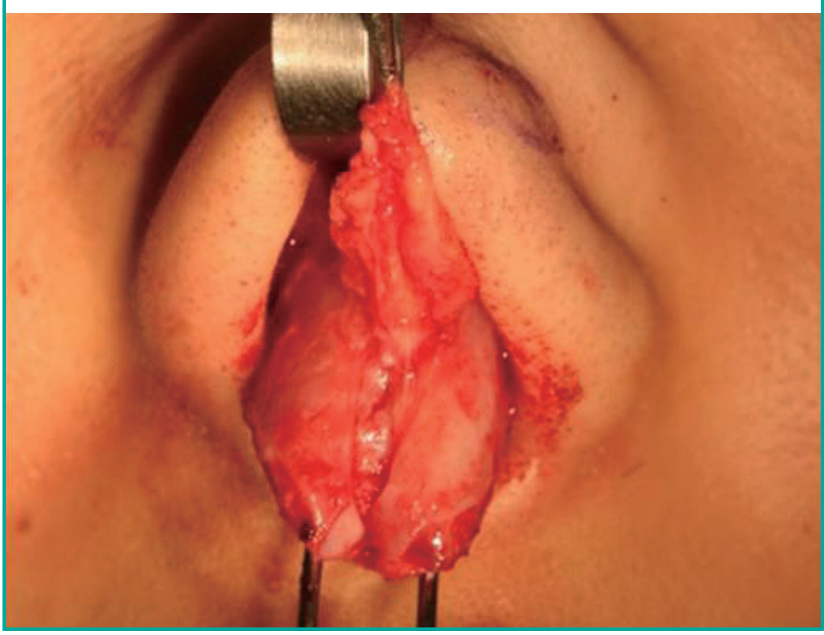

Fig. 10. Photographs of case 1 patient

(A) Preoperative frontal view when smiling. (B) Twenty-month postoperative frontal view when smiling.
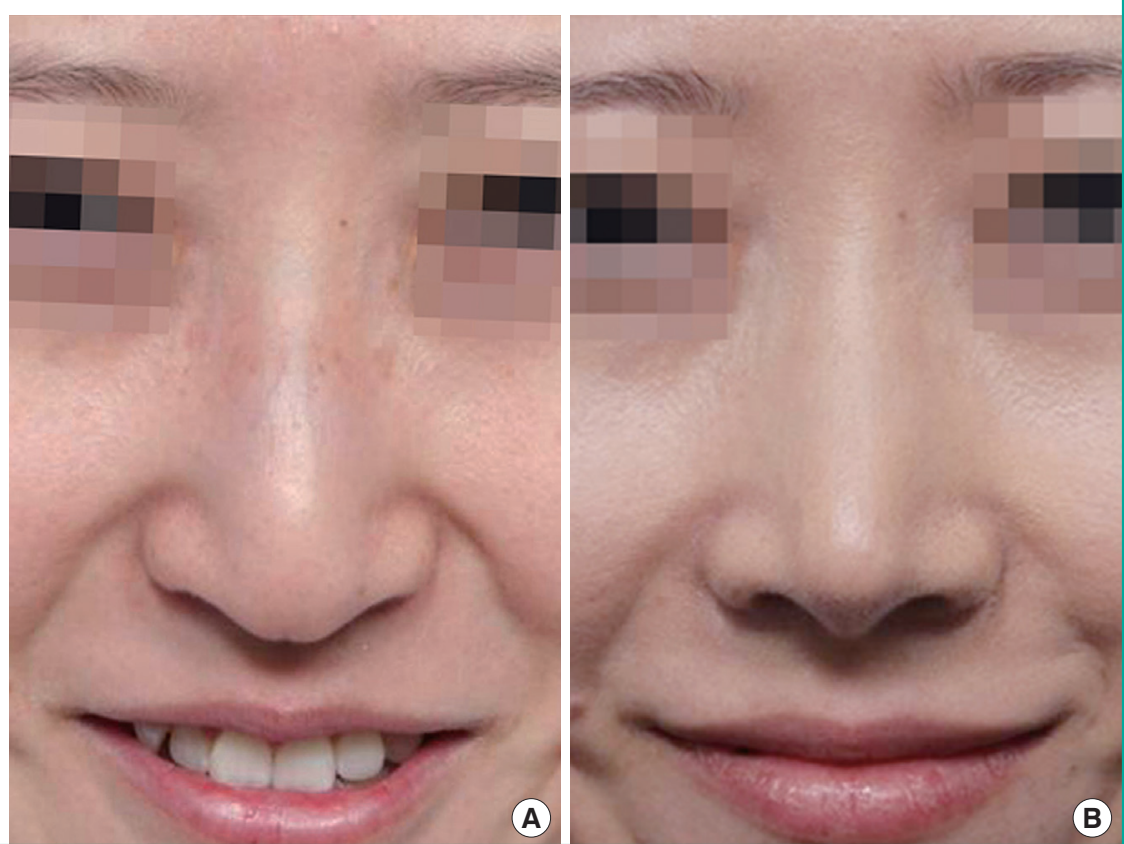


\section{Fig. 11. Photographs of case 2 patient}

(A) Preoperative lateral view when smiling.

(B) Two-month postoperative lateral view when smiling.
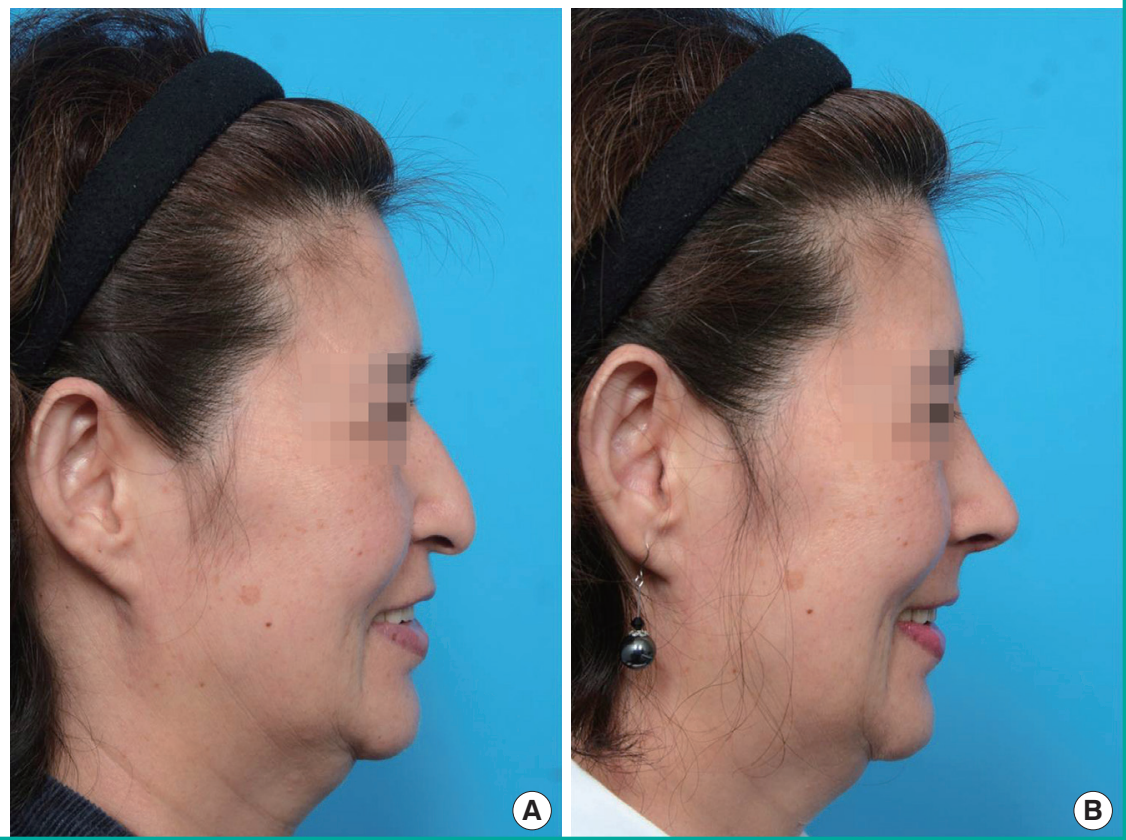

incisions were performed.

\section{Case 1}

A 28-year-old woman complained of a convex nasal dorsum with a mild hump, low radix, and drooping tip with a long nose. Her drooping tip worsened when she smiled. She underwent a columellar strut with cymba conchal cartilage, nasal tip cephalic rotation using the turn-up cartilage flap of the cephalic portion of the lateral crura, humpectomy with lateral osteotomy, augmentation of the radix with crushed conchal cartilage, and perialar and columellar base augmentation (Fig. 10).

\section{Case 2}

A 52-year-old woman was not satisfied with her convex long nose with a drooping tip. She also had a nasal dorsal hump, low radix, and worsening drooping tip when she smiled. She underwent distal septum excision, batten graft for tip support, nasal tip cephalic rotation using the turn-up cartilage flap of the cephalic portion of the lateral crura, humpectomy with lateral osteotomy, and augmentation of the radix with crushed septal cartilage (Fig. 11).

\section{CONCLUSIONS}

A drooping tip has multiple causes that can be classified according to their mechanisms. The static causes result from the position and shape of the nasal cartilages, while the dynamic causes are a result of the muscles that affect the tip. East Asians usually have a small medial crus of alar cartilage and a more mobile tip caused by weak tip support. They also have additional static components such as thick nasal skin. Further, dynamic components including the depressor septi nasi muscle, levator labii superioris alaeque nasi muscle, and associated alar base depression are more prominent. Along with correcting the static and dynamic components, ancillary procedures such as debulking the bulbous tip soft tissue, redundant nasal skin excision, columellar base, and perialar augmentations are essential for obtaining optimum results.

To achieve and preserve adequate correction of the drooping tip in East Asian long noses, multifactorial approaches to static and dynamic aspects along with ancillary causes should be considered and corrected simultaneously.

\section{REFERENCES}

1. Byrd HS, Hobar PC. Rhinoplasty: a practical guide for surgical planning. Plast Reconstr Surg 1993;91:642-54.

2. Arregui JS, Elejalde MV, Regalado J, et al. Dynamic rhinoplasty for the plunging nasal tip: functional unity of the inferior third of the nose. Plast Reconstr Surg 2000;106:1624-9.

3. Rohrich RJ, Huynh B, Muzaffar AR, et al. Importance of the depressor septi nasi muscle in rhinoplasty: anatomic study and clinical application. Plast Reconstr Surg 2000;105:37683.

4. Jeong JY, Oh SH, Lee SR, et al. Muscular system of depressor septi nasi: anatomical study and clinical application. J 
Korean Soc Aesthetic Plast Surg 2009; 15:49-54.

5. Foda HM. Management of the droopy tip: a comparison of three alar cartilage-modifying techniques. Plast Reconstr Surg 2003;112:1408-17.

6. Behmand RA, Ghavami A, Guyuron B. Nasal tip sutures part I: the evolution. Plast Reconstr Surg 2003;112:1125-9.

7. Guyuron B, Behmand RA. Nasal tip sutures part II: the interplays. Plast Reconstr Surg 2003;112:1130-45.

8. Tebbetts JB. Shaping and positioning the nasal tip without structural disruption: a new, systematic approach. Plast Reconstr Surg 1994;94:61-77.
9. Boccieri A. Mini spreader grafts: a new technique associated with reshaping of the nasal tip. Plast Reconstr Surg 2005; 116:1525-34.

10. Anderson JR. A reasoned approach to nasal base surgery. Arch Otolaryngol 1984;110:349-58.

11. Gunter JP, Landecker A, Cochran CS. Frequently used grafts in rhinoplasty: nomenclature and analysis. Plast Reconstr Surg 2006;118:14e-29e.

12. Ko BM, Kang SY, Yoo YC, et al. Anatomical nasal tip plasty with autogenous cartilage graft. J Korean Soc Plast Reconstr Surg 2002;29:17-22. 